\title{
Wie weit soll der Blutdruck bei Typ-2-Diabetikern gesenkt werden?
}

\begin{abstract}
Bedeutende Studien zur antihypertensiven Therapie haben eine intensive Diskussion um den Zielblutdruck bei Typ-2-Diabetikern ausgelöst und zu Änderungen in den Leitlinien der Fachgesellschaften geführt. Einige Mängel sind aber unverkennbar: Es geht fast ausschließlich um die Frage, ob der systolische Druck unter $140 \mathrm{mmHg}$ oder unter $130 \mathrm{mmHg}$ gesenkt werden soll, wobei dem Zwischenbereich wenig Beachtung geschenkt wird. Zudem werden alle Endpunkte insgesamt betrachtet und die Nebenwirkungen bei unterschiedlichen Zielwerten bleiben unberücksichtigt.
\end{abstract}

- Die Autoren suchten nach randomisierten Studien bei Typ-2-Diabetikern oder Patienten mit gestörter Glukosetoleranz, die mindestens 100 Patienten einbezogen haben, ein Jahr dauerten und unterschiedliche Zielblutdruckwerte anstrebten. 13 Studien mit 37736 Patienten und einer Beobachtungsdauer bis zu 4,8 Jahren erfüllten diese Kriterien. Die Ergebnisse sind statistisch gut abgesichert.

Im Vergleich mit einer Blutdrucksenkung unter $140 \mathrm{mmHg}$ senkt ein Zielwert unter $135 \mathrm{mmHg}$ die Gesamtmor-

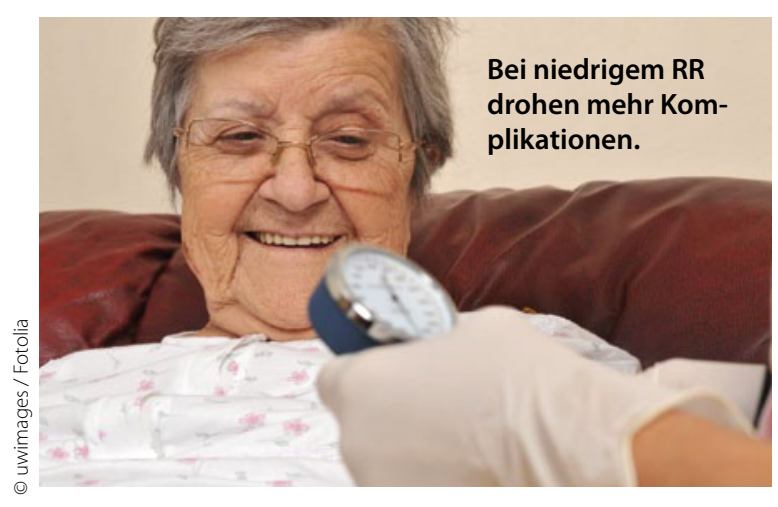

verhindert deutlich mehr Schlaganfälle. Auch bei den mikrovaskulären renalen, retinalen und neuralen Endpunkten bringt die Senkung unter $135 \mathrm{mmHg}$ keinen Vorteil. Im Vergleich zum Zielwert unter $140 \mathrm{mmHg}$ nehmen schwere Nebenwirkungen (bedrohliche Folgen, permanente Behinderung oder Studienabbruch) bei Sen-

talität, die kardiovaskuläre Mortalität, die Zahl an Herzinfarkten, Herzinsuffizienz und Schlaganfällen. Bei einer Senkung unter $130 \mathrm{mmHg}$ ergibt sich kein Zusatznutzen, bei einigen Endpunkten deutet sich sogar eine Tendenz zur Verschlechterung an. Einzige Ausnahme: Eine weitere Senkung unter $130 \mathrm{mmHg}$

\section{Kommentar}

Die vorliegende Metaanalyse beantwortet Fragen, die bisher offen blieben oder nicht bearbeitet wurden. Die Aussagen sind differenzierter, erleichtern aber nicht unbedingt generelle Empfehlungen. Denn zur Verhinderung diabetischer Komplikationen an den verschiedenen Organen gibt es keinen einheitlichen Zielblutdruck. Obwohl eine
Blutdrucksenkung unter 130, ja sogar unter $120 \mathrm{mmHg}$ Schlaganfälle verhindert, trifft dies nicht für andere kardiale, retinale und - in dieser Analyse - renale Komplikationen $z u$. Es gibt also eine Heterogenität des Zielblutdrucks für die verschiedenen Organe. Nur für die schweren Nebenwirkungen gilt weiterhin: je stärker die Blutdrucksenkung, desto häufiger.

Man kann als generelle Konsequenz für Typ2-Diabetiker einen systolischen Zielblutdruck unter 135 statt unter $140 \mathrm{mmHg}$ empfehlen, wenn man die Zunahme schwerer Nebenwirkungen um 20\% in Kauf nehmen will. Wer wirklich glaubt prognostizieren zu können, dass sein Patient in besonderem Maß durch Schlaganfall gefährdet ist, kann den Blutdruck auch unter $130 \mathrm{mmHg}$ senken, allerdings auf Kosten von mehr Nebenwirkungen und ohne Zusatznutzen auf andere

Organsysteme.

H. HOLZGREVE =

- S. Bangalore et al.

Blood pressure targets in subjects with type

2 diabetes mellitus/impaired fasting glucose.

Circulation 21 (2011) 2799-2810 